\title{
Observation of Exclusive Deeply Virtual Compton Scattering in Polarized Electron Beam Asymmetry Measurements
}

\author{
S. Stepanyan
}

Angela Biselli

Fairfield University, abiselli@fairfield.edu

CLAS Collaboration

Follow this and additional works at: https://digitalcommons.fairfield.edu/physics-facultypubs

Copyright American Physical Society Publisher final version available at http://prl.aps.org/pdf/

PRL/v87/i18/e182002

\section{Peer Reviewed}

\section{Repository Citation}

Stepanyan, S.; Biselli, Angela; and CLAS Collaboration, "Observation of Exclusive Deeply Virtual Compton Scattering in Polarized Electron Beam Asymmetry Measurements" (2001). Physics Faculty Publications. 104.

https://digitalcommons.fairfield.edu/physics-facultypubs/104

\section{Published Citation}

S. Stepanyan et al. [CLAS Collaboration], "Observation of Exclusive Deeply Virtual Compton Scattering in Polarized Electron Beam Asymmetry Measurements", Physical Review Letters 87.18 (2001) DOI: 10.1103/

PhysRevLett.87.182002

This item has been accepted for inclusion in DigitalCommons@Fairfield by an authorized administrator of DigitalCommons@Fairfield. It is brought to you by DigitalCommons@Fairfield with permission from the rightsholder(s) and is protected by copyright and/or related rights. You are free to use this item in any way that is permitted by the copyright and related rights legislation that applies to your use. For other uses, you need to obtain permission from the rights-holder(s) directly, unless additional rights are indicated by a Creative Commons license in the record and/or on the work itself. For more information, please contact digitalcommons@fairfield.edu. 


\title{
Observation of Exclusive Deeply Virtual Compton Scattering in Polarized Electron Beam Asymmetry Measurements
}

S. Stepanyan, ${ }^{1,2}$ V. D. Burkert, ${ }^{2}$ L. Elouadrhiri, ${ }^{1,2}$ G. S. Adams, ${ }^{28}$ E. Anciant, ${ }^{7}$ M. Anghinolfi, ${ }^{15}$ B. Asavapibhop, ${ }^{20}$ G. Audit, ${ }^{7}$ T. Auger, ${ }^{7}$ H. Avakian, ${ }^{14}$ J. Ball, ${ }^{3}$ S. Barrow, ${ }^{13}$ M. Battaglieri,,${ }^{15}$ K. Beard, ${ }^{18}$ M. Bektasoglu, ${ }^{25}$ P. Bertin, ${ }^{9}$ N. Bianchi, ${ }^{14}$ A. Biselli, ${ }^{28}$ S. Boiarinov, ${ }^{17}$ B. E. Bonner, ${ }^{29}$ S. Bouchigny, ${ }^{2,26}$ D. Branford, ${ }^{11}$ W. K. Brooks, ${ }^{2}$ J. R. Calarco, ${ }^{22}$ D. S. Carman, ${ }^{24}$ B. Carnahan, ${ }^{6}$ L. Ciciani, ${ }^{25}$ P. L. Cole,${ }^{32,2}$ A. Coleman, ${ }^{35, *}$ D. Cords, ${ }^{2}$ P. Corvisiero, ${ }^{15}$ D. Crabb, ${ }^{33}$ H. Crannell, ${ }^{6}$ J. Cummings, ${ }^{28}$ P. V. Degtiarenko, ${ }^{2}$ H. Denizli, ${ }^{27}$ L.C. Dennis, ${ }^{13}$ E. De Sanctis, ${ }^{14}$ R. DeVita, ${ }^{15}$ K. V. Dharmawardane, ${ }^{25}$ C. Djalali, ${ }^{31}$ G. E. Dodge, ${ }^{25}$ D. Doré, ${ }^{7}$ D. Doughty, ${ }^{1,2}$ P. Dragovitsch, ${ }^{13}$ S. Dytman, ${ }^{27}$ M. Eckhause, ${ }^{35}$ H. Egiyan, ${ }^{35}$ K. S. Egiyan, ${ }^{36}$ A. Empl, ${ }^{28}$ R. Fatemi, ${ }^{33}$ R. J. Feuerbach, ${ }^{5}$ J. Ficenec, ${ }^{34}$ K. Fissum, ${ }^{21}$ T. A. Forest, ${ }^{25}$ A. P. Freyberger, ${ }^{25}$ H. Funsten, ${ }^{35}$ S. Gaff, ${ }^{10}$ M. Gai, ${ }^{8}$ M. Garçon, ${ }^{7}$ G. Gavalian,,${ }^{36}$, S. Gilad, ${ }^{21}$ G. P. Gilfoyle, ${ }^{30}$ K. Giovanetti, ${ }^{18}$ P. Girard, ${ }^{31}$ K. A. Griffioen, ${ }^{35}$ M. Guidal,,${ }^{26}$ M. Guillo, ${ }^{31}$ V. Gyurjyan, ${ }^{2}$ C. Hadjidakis, ${ }^{26}$ J. Hardie, ${ }^{1,2}$ D. Heddle, ${ }^{1,2}$ F. W. Hersman, ${ }^{22}$ K. Hicks, ${ }^{24}$ R. S. Hicks, ${ }^{20}$ M. Holtrop, ${ }^{22}$ J. Hu, ${ }^{28}$ C. E. Hyde-Wright,${ }^{25}$ M. M. Ito, ${ }^{2}$ D. Jenkins,${ }^{34}$ K. Joo, ${ }^{33, \$}$ J. Kelley, ${ }^{10}$ M. Khandaker, ${ }^{23}$ D. H. Kim, ${ }^{19}$ K. Kim, ${ }^{19}$ K. Y. Kim, ${ }^{27}$ W. Kim, ${ }^{19}$ A. Klein, ${ }^{25}$ F. J. Klein, ${ }^{2}$ M. Klusman, ${ }^{28}$ M. Kossov, ${ }^{17}$ L. H. Kramer, ${ }^{12,2}$ S. E. Kuhn, ${ }^{25}$ J. M. Laget, ${ }^{7}$ D. Lawrence, ${ }^{20}$ A. Longhi, ${ }^{6}$ K. Lukashin, ${ }^{32,2,}{ }^{8}$ J. J. Manak, ${ }^{2, \|}$ C. Marchand, ${ }^{7}$ S. McAleer, ${ }^{13}$ J. McCarthy, ${ }^{33}$ J. W. C. McNabb, ${ }^{5}$ B. A. Mecking, ${ }^{2}$ M. D. Mestayer, ${ }^{2}$ C. A. Meyer, ${ }^{5}$ K. Mikhailov, ${ }^{17}$ R. Minehart, ${ }^{33}$ M. Mirazita, ${ }^{14}$ R. Miskimen, ${ }^{20}$ L. Morand, ${ }^{7}$ V. Muccifora, ${ }^{14}$ J. Mueller, ${ }^{27}$ G. S. Mutchler, ${ }^{29}$ J. Napolitano, ${ }^{28}$ S. Nelson, ${ }^{10}$ S. Niccolai, ${ }^{16}$ G. Niculescu, ${ }^{24}$ I. Niculescu, ${ }^{16}$ R. A. Niyazov, ${ }^{25}$ A. Opper, ${ }^{24}$ G. O'Rielly, ${ }^{16}$ J. T. O’Brien, ${ }^{6}$ K. Park, ${ }^{19}$ E. Pasyuk, ${ }^{3}$ G. A. Peterson, ${ }^{20}$ S. Philips,${ }^{16}$ N. Pivnyuk, ${ }^{17}$ D. Pocanic, ${ }^{33}$ O. Pogorelko, ${ }^{17}$ E. Polli, ${ }^{14}$ I. Popa,${ }^{16}$ S. Pozdniakov,,${ }^{17}$ B. M. Preedom, ${ }^{31}$ J. W. Price, ${ }^{31}$ D. Protopopescu, ${ }^{22}$ L. M. Qin, ${ }^{25}$ B. A. Raue, ${ }^{12,2}$ A. R. Reolon, ${ }^{14}$ G. Riccardi, ${ }^{13}$ G. Ricco, ${ }^{15}$ M. Ripani, ${ }^{15}$ B. G. Ritchie, ${ }^{3}$ F. Ronchetti, ${ }^{14}$ P. Rossi, ${ }^{14}$ D. Rowntree, ${ }^{21}$ P. D. Rubin, ${ }^{30}$ F. Sabatié,,${ }^{7,25}$ K. Sabourov, ${ }^{10}$ C. W. Salgado, ${ }^{23,2}$ V. Sapunenko, ${ }^{15}$ R. A. Schumacher, ${ }^{5}$ V. Serov, ${ }^{17}$ Y. G. Sharabian, ${ }^{36,2}$ J. Shaw, ${ }^{20}$ S. Simionatto, ${ }^{16}$ A. Skabelin, ${ }^{21}$ E. S. Smith, ${ }^{2}$ L. C. Smith, ${ }^{33}$ D. I. Sober, ${ }^{6}$ A. Stavinsky, ${ }^{17}$ P. Stoler,${ }^{28}$ I. I. Strakovsky, ${ }^{16}$ R. Suleiman, ${ }^{21}$ M. Taiuti, ${ }^{15}$ S. Taylor, ${ }^{29}$ D. Tedeschi, ${ }^{31,2}$ R. Thompson, ${ }^{27}$ L. Todor, ${ }^{5}$ M. F. Vineyard, ${ }^{30}$ A. Vlassov, ${ }^{17}$ K. Wang, ${ }^{33}$ H. Weller, ${ }^{10}$ L. B. Weinstein, ${ }^{25}$ R. Welsh, ${ }^{35}$ D. P. Weygand, ${ }^{2}$ S. Whisnant,${ }^{31}$ E. Wolin, ${ }^{2}$ A. Yegneswaran, ${ }^{2}$ J. Yun, ${ }^{25}$ J. Zhao, ${ }^{21}$ B. Zhang, ${ }^{21}$ and Z. Zhou ${ }^{21}$

(CLAS Collaboration)

\author{
${ }^{1}$ Christopher Newport University, Newport News, Virginia 23606 \\ ${ }^{2}$ Thomas Jefferson National Accelerator Facility, Newport News, Virginia 23606 \\ ${ }^{3}$ Arizona State University, Department of Physics and Astronomy, Tempe, Arizona 85287 \\ ${ }^{4}$ University of California at Los Angeles, Department of Physics and Astronomy, Los Angeles, CA 90095 \\ ${ }^{5}$ Carnegie Mellon University, Department of Physics, Pittsburgh, Pennsylvania 15213 \\ ${ }^{6}$ Catholic University of America, Department of Physics, Washington, D.C. 20064 \\ ${ }^{7}$ CEA Saclay, DAPNIA-SPhN, F91191 Gif-sur-Yvette Cedex, France \\ ${ }^{8}$ University of Connecticut, Physics Department, Storrs, Connecticut 06269 \\ ${ }^{9}$ University Blaise Pascal/IN2P3, Aubiere Cedex, France \\ ${ }^{10}$ Duke University, Physics Department, Durham, North Carolina 27706 \\ ${ }^{11}$ Edinburgh University, Department of Physics and Astronomy, Edinburgh EH9 3JZ, United Kingdom \\ ${ }^{12}$ Florida International University, Department of Physics, Miami, Florida 33199 \\ ${ }^{13}$ Florida State University, Department of Physics, Tallahassee, Florida 32306 \\ ${ }^{14}$ Istituto Nazionale di Fisica Nucleare, Laboratori Nazionali di Frascati, C.P. 13, 00044 Frascati, Italy \\ ${ }^{15}$ Istituto Nazionale di Fisica Nucleare, Sezione di Genova e Dipartimento di Fisica dell'Universita, 16146 Genova, Italy \\ ${ }^{16}$ The George Washington University, Department of Physics, Washington, D.C. 20052 \\ ${ }^{17}$ Institute of Theoretical and Experimental Physics, Moscow, 117259, Russia \\ ${ }^{18}$ James Madison University, Department of Physics, Harrisonburg, Virginia 22807 \\ ${ }^{19}$ Kyungpook National University, Department of Physics, Taegu 702-701, South Korea \\ ${ }^{20}$ University of Massachusetts, Department of Physics, Amherst, Massachusetts 01003 \\ ${ }^{21}$ MIT-Bates Linear Accelerator, Middleton, Massachusetts 01949 \\ ${ }^{22}$ University of New Hampshire, Department of Physics, Durham, New Hampshire 03824 \\ ${ }^{23}$ Norfolk State University, Norfolk, Virginia 23504 \\ ${ }^{24}$ Ohio University, Department of Physics, Athens, Ohio 45701 \\ ${ }^{25}$ Old Dominion University, Department of Physics, Norfolk, Virginia 23529 \\ ${ }^{26}$ Institut de Physique Nucleaire d'Orsay, IN2P3, BP 1, 91406 Orsay, France
}




\author{
${ }^{27}$ University of Pittsburgh, Department of Physics, Pittsburgh, Pennsylvania 15260 \\ ${ }^{28}$ Rensselaer Polytechnic Institute, Department of Physics, Troy, New York 12181 \\ ${ }^{29}$ Rice University, T.W. Bonner Nuclear Laboratory, Houston, Texas 77005-1892 \\ ${ }^{30}$ University of Richmond, Department of Physics, Richmond, Virginia 23173 \\ ${ }^{31}$ University of South Carolina, Department of Physics, Columbia, South Carolina 29208 \\ ${ }^{32}$ University of Texas, Department of Physics, El Paso, Texas 79968 \\ ${ }^{33}$ University of Virginia, Department of Physics, Charlottesville, Virginia 22903 \\ ${ }^{34}$ Virginia Polytechnic Institute and State University, Department of Physics, Blacksburg, Virginia 24061 \\ ${ }^{35}$ College of William and Mary, Department of Physics, Williamsburg, Virginia 23185 \\ ${ }^{36}$ Yerevan Physics Institute, 375036 Yerevan, Armenia \\ (Received 17 July 2001; published 10 October 2001)
}

\begin{abstract}
We report the first results of the beam-spin asymmetry measured in the reaction $\vec{e} p \rightarrow e p \gamma$ at a beam energy of $4.25 \mathrm{GeV}$. A large asymmetry with a $\sin \phi$ modulation is observed, as predicted for the interference term of deeply virtual compton scattering (DVCS) and the Bethe-Heitler process. The amplitude of this modulation is $\alpha=0.202 \pm 0.028$. In leading-order and leading-twist perturbative QCD, the $\alpha$ is directly proportional to the imaginary part of the DVCS amplitude.
\end{abstract}

DOI: 10.1103/PhysRevLett.87.182002

PACS numbers: 13.60.Fz, 14.20.Dh, 24.85.+p

High-energy lepton nucleon scattering experiments revealed the quark structure of the nucleon and were employed to measure the longitudinal quark momentum distributions as well as the quark spin distributions. The recently developed formalism [1-4] for the QCD description of deeply exclusive leptoproduction reactions introduces generalized parton distributions (GPDs). They carry new information about the dynamical degrees of freedom inside the nucleon not accessible in inclusive experiments. It has been shown that in the Bjorken scaling regime (high photon virtuality $Q^{2}$, high-energy transfer at fixed Bjorken scaling variable $x_{B}$ ) the scattering amplitude for exclusive processes can be factorized into a hardscattering part (exactly calculable in perturbative QCD) and a nucleon structure part parametrized via GPDs. This is called the "handbag approximation," depicted in Fig. 1a. In addition to the dependence on the parton momentum fraction $x$, GPDs depend on two more parameters, the skewedness $\xi$, and the momentum transfer $t$ to the baryonic system. In the Bjorken regime $\xi \rightarrow x_{B} /\left(2-x_{B}\right)$, and is related to the momentum imbalance between the struck quark and the quark that is put back into the final state baryon. The momentum transfer $t$, which can be varied independently in exclusive processes, measures the transverse momentum transfer between interacting quarks. The GPDs reduce to the longitudinal parton distributions in the limit $\xi \rightarrow 0, t \rightarrow 0$.

Deeply exclusive experiments probe the nucleon wave function at the quark-gluon level, expressed in terms of two spin-dependent and two spin-independent GPDs. Deeply virtual compton scattering (DVCS), i.e., electroproduction

$$
\frac{d^{4} \sigma^{+}}{d Q^{2} d x_{B} d t d \phi}-\frac{d^{4} \sigma^{-}}{d Q^{2} d x_{B} d t d \phi} \propto \operatorname{Im}\left(T^{\mathrm{DVCS}}\right) T^{\mathrm{BH}} \propto
$$

Here $a$ and $b$ are independent of $\phi$, the angle between the lepton and hadron planes, and $\tilde{M}^{1,1}$ and $\tilde{M}^{0,1}$ are the helicity amplitudes for transverse and longitudinal virtual photons, respectively. The cross section for positive (nega- of photons from nucleons in the Bjorken regime, is most suitable for studying GPDs at moderately high energies. While exclusive meson production requires high energies and high photon virtualities to reach the Bjorken regime, DVCS may access the GPDs already at $Q^{2}$ as low as $1(\mathrm{GeV} / c)^{2}[5,6]$.

In this Letter we present the first observation of the fully exclusive DVCS signal in the beam-spin asymmetry measured in the reaction $\vec{e} p \rightarrow e p \gamma$. The measurement was performed using a $4.25 \mathrm{GeV}$ longitudinally polarized electron beam and the CEBAF Large Acceptance Spectrometer (CLAS) [7] at the Thomas Jefferson National Accelerator Facility. Observation of the beam-spin asymmetry in semiexclusive production of high-energy photons has been reported recently by HERMES collaboration [8], and cross section measurements of DVCS by H1 Collaboration at DESY at very small $x_{B}$ [9], which is complementary to the valence quark regime of this measurement.

The experiments measure the DVCS contribution through its interference with the Bethe-Heitler (BH) process. In contrast to DVCS, where the photon is emitted from the nucleon, $\mathrm{BH}$ photons are emitted from the incoming or scattered electrons (Fig. 1). While the $\mathrm{BH}$ cross section in most of the kinematic region is much larger than the DVCS cross section, the interference between the two amplitudes boosts the effect of DVCS and produces a large cross section difference for electron helicities aligned or antialigned with the electron beam. In this difference, the large helicity-independent $\mathrm{BH}$ contribution drops out, and only the helicity-dependent interference term remains [10]:

$$
a \operatorname{Im} \tilde{M}^{1,1} \sin \phi+b \operatorname{Im} \tilde{M}^{0,1} \sin 2 \phi+O\left(\frac{1}{Q^{2}}\right)+\ldots
$$

tive) electron-beam helicity is denoted with $\sigma^{+}\left(\sigma^{-}\right)$. This difference measures directly the imaginary part of the DVCS amplitude, since the BH amplitude can 


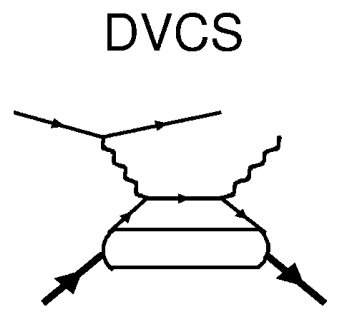

(a)

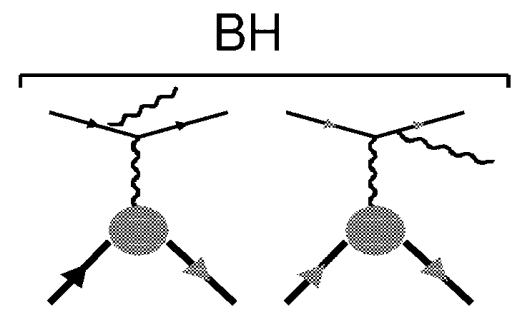

(b)
FIG. 1. Feynman diagrams for DVCS and Bethe-Heitler processes contributing to the amplitude of $e p \rightarrow e p \gamma$ scattering.

be calculated exactly. In leading order, only $\tilde{M}^{1,1}$ contributes [10].

Experimentally it is much simpler to measure an asymmetry $A=\left(d^{4} \sigma^{+}-d^{4} \sigma^{-}\right) /\left(d^{4} \sigma^{+}+d^{4} \sigma^{-}\right)$rather than a cross section difference. This asymmetry gives the relative strength of the relevant amplitudes and has a more complex dependence on $\phi$ than the cross section difference, due to $\phi$ terms in the denominator. In the Bjorken limit, however, the $\phi$ dependence of the denominator is expected to be small.

In this analysis, we used CLAS electroproduction data taken in March 1999. Scattering of $4.25 \mathrm{GeV}$ longitudinally polarized electrons from a liquid hydrogen target was studied in a wide range of kinematics. The analyzed event sample corresponds to an integrated luminosity of $1.3 \mathrm{fb}^{-1}$.

The reaction $e p \rightarrow e p \gamma$ is identified by analyzing the missing-mass squared distributions in the reaction $e p \rightarrow$ epX: $\quad M_{x}^{2}=\left(\nu+M-E_{p}\right)^{2}-\left(\vec{q}-\vec{P}_{p}\right)^{2}$, where $\nu$ and $\vec{q}$ are the virtual photon energy and momentum, $E_{p}$ and $\vec{P}_{p}$ are the energy and momentum of the recoil proton, and $M$ is the mass of the proton.

The main background to the single photon final state is from $\pi^{0}$ production. At large missing energy, the missingmass resolution of CLAS is not sufficient to separate single photons and $\pi^{0}$ s event-by-event. The number of single photon events was determined using a fitting technique that analyzes the line shape of the $M_{x}^{2}$ distributions. As a fit function the sum of two Gaussians and a third order polynomial is used:

$$
\begin{aligned}
F\left(M_{x}^{2}\right)= & N_{\gamma} e^{-\left(M_{x}^{2}-M_{\gamma}^{2}\right)^{2} / 2 \sigma_{\gamma}^{2}} \\
& +N_{\pi^{0}} e^{-\left(M_{x}^{2}-M_{\pi}^{2}\right)^{2} / 2 \sigma_{\pi}^{2}} \\
& +\sum_{j=0}^{3} P_{j}\left(M_{x}^{2}\right)^{j} .
\end{aligned}
$$

The Gaussians represent the $M_{x}^{2}$ distributions for single photon and single pion final states. The polynomial is used to model the smooth background that arises from radiative processes. The parameters $M_{\gamma}^{2}$ and $\sigma_{\gamma}^{2}$ are determined

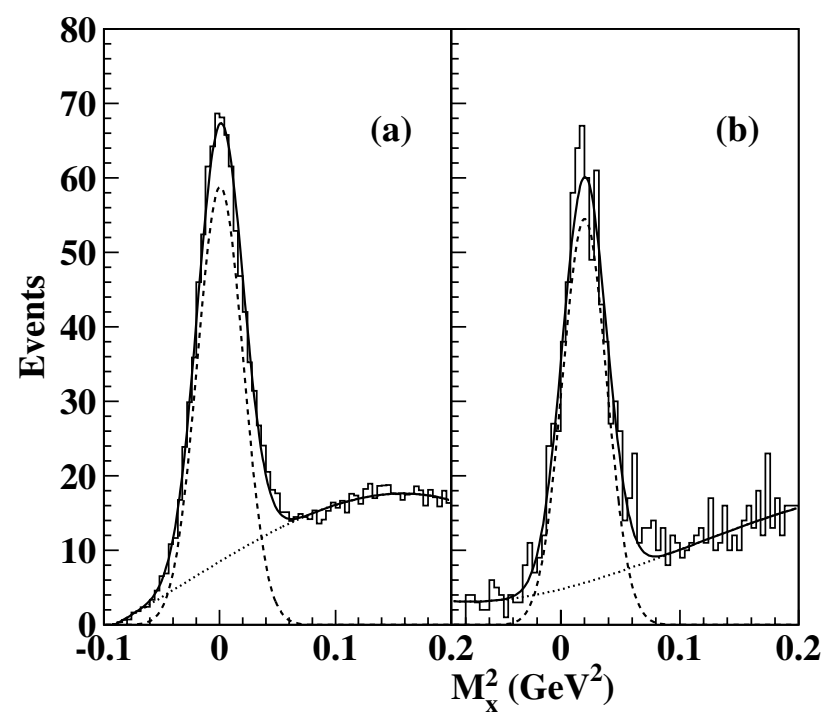

FIG. 2. Missing-mass squared distribution of the detected (ep) system for (a) $e p \rightarrow e p \gamma$ and (b) $e p \rightarrow e p \pi^{0}$. In each plot the solid line is the fit to the sum of a Gaussian and the third order polynomial distribution. The dashed curve corresponds to the Gaussian function and the dotted curve represents the polynomial function.

from the fit to the $M_{x}^{2}$ distribution of selected Bethe-Heitler events (Fig. 2a). For $M_{\pi}^{2}$ and $\sigma_{\pi}^{2}$, the results of a similar fit to the $M_{x}^{2}$ distribution of selected events with final state $e p \rightarrow e p \gamma \gamma$ are used (Fig. 2b). In these events both photons are detected in the CLAS electromagnetic calorimeter; they have an invariant mass near the $\pi^{0}$ mass. In Fig. 2 the curves represent the fit to a sum of the Gaussian and a third order polynomial. The samples of $\mathrm{BH}$ and $\pi^{0}$ events were selected in the same kinematical range as the main sample for DVCS studies.

The $M_{x}^{2}$ distributions are analyzed in eleven $\phi$ bins. The final fit to the $M_{x}^{2}$ distributions with the function in Eq. (2) is performed separately for each helicity state and for the sum. In the fit only two parameters are adjusted: the number of single photon $\left(N_{\gamma}\right)$ and the number of $\pi^{0}\left(N_{\pi^{0}}\right)$ events. For each $\phi$ bin the shape of the background was fixed from a fit to the points in the summed $M_{x}^{2}$ distribution that excluded the $M_{x}^{2}$ range from $-0.07 \mathrm{GeV}^{2}$ to $0.08 \mathrm{GeV}^{2}$. The relative magnitude of the background for distributions at positive and negative helicities is found from the fit to the same $M_{x}^{2}$ range as for the sum.

Figure 3 shows a typical fit result for the sum of two helicities at $\phi=90^{\circ}$. The solid line corresponds to the fitted function $F\left(M_{x}^{2}\right)$, the dashed line represents the Gaussian for the missing photon, and the dash-dotted line is the Gaussian for the missing $\pi^{0}$. The dotted line represents the polynomial background. In this bin the number of photon and pion events are $N_{\gamma}=4201 \pm 109$ and $N_{\pi^{0}}=$ $2010 \pm 101$, respectively. The $\chi^{2}$ per degree of freedom of the fit is 1.8. Similar results have been obtained for all kinematical bins. 


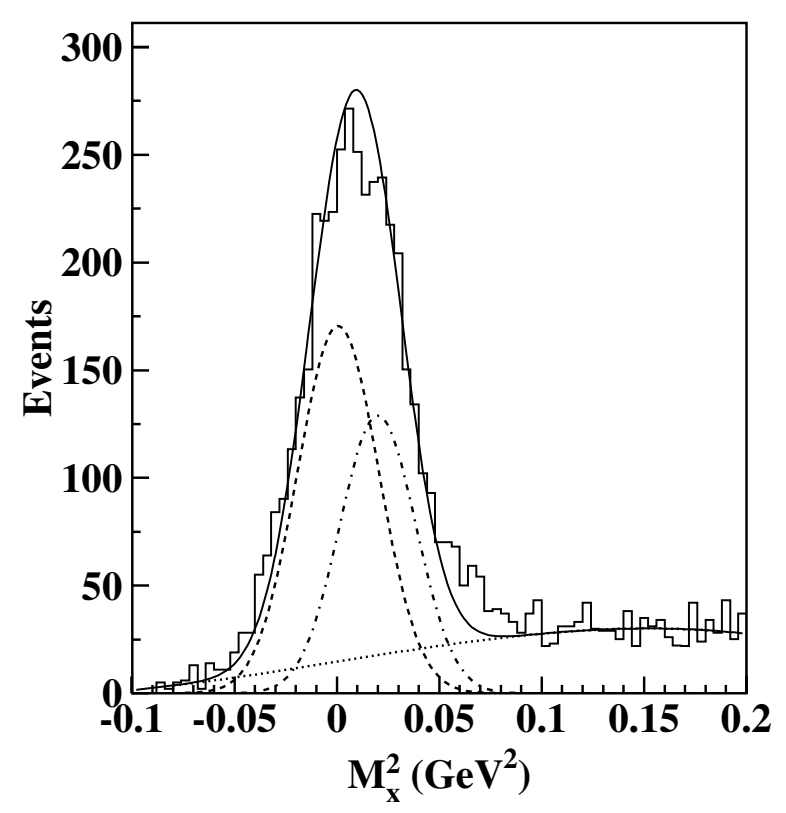

FIG. 3. Missing-mass squared distribution for the reaction $e p \rightarrow e p X$. Events are integrated in the range of $\phi$ from $70^{\circ}$ to $110^{\circ}$. The curves are described in the text.

The fitted number of single photon events are used to calculate the beam-spin asymmetry as

$$
A=\frac{1}{P_{e}} \frac{\left(N_{\gamma}^{+}-N_{\gamma}^{-}\right)}{\left(N_{\gamma}^{+}+N_{\gamma}^{-}\right)},
$$

where $P_{e}$ is the beam polarization, and $N_{\gamma}^{+(-)}$is the number of $e p \rightarrow e p \gamma$ events at positive (negative) beam helicity. The average beam polarization, $P_{e}=70 \%$, was measured using Møller scattering.

In Fig. 4 we present our main result, the $\phi$ dependence of $A$. Data in each $\phi$ bin are integrated in the range of $Q^{2}$ from $1.00(\mathrm{GeV} / c)^{2}$ to $1.75(\mathrm{GeV} / c)^{2}$ and $-t$ from $0.1(\mathrm{GeV} / c)^{2}$ to $0.3(\mathrm{GeV} / c)^{2}$. The error bars shown are statistical. Most of the systematic uncertainties related to the experiment do not contribute to $A$. Only the error in the measurement of beam polarization, $\pm 1.65 \%$, remains. There is also a systematic error in the calculation of $N_{\gamma}$ due to the determination of the mean and standard deviation of the Gaussian functions for the photon and pion missing-mass squared distributions, and also due to the fit procedure to the $M_{x}^{2}$ distributions. These errors are defined as a deviation of $A$ from its central value, when the mean and standard deviation of Gaussians are shifted within their errors, and when different fit techniques were used (see [12] for details).

The data points are fitted with the function $A(\phi)=$ $\alpha \sin \phi+\beta \sin 2 \phi$. The fitted parameters are $\alpha=$ $0.202 \pm 0.028^{\text {stat }} \pm 0.013^{\text {syst }}$ and $\beta=-0.024 \pm$ $0.021^{\text {stat }} \pm 0.009^{\text {syst }}$. In the Bjorken regime $\beta$ should vanish, leaving only the contribution from transverse

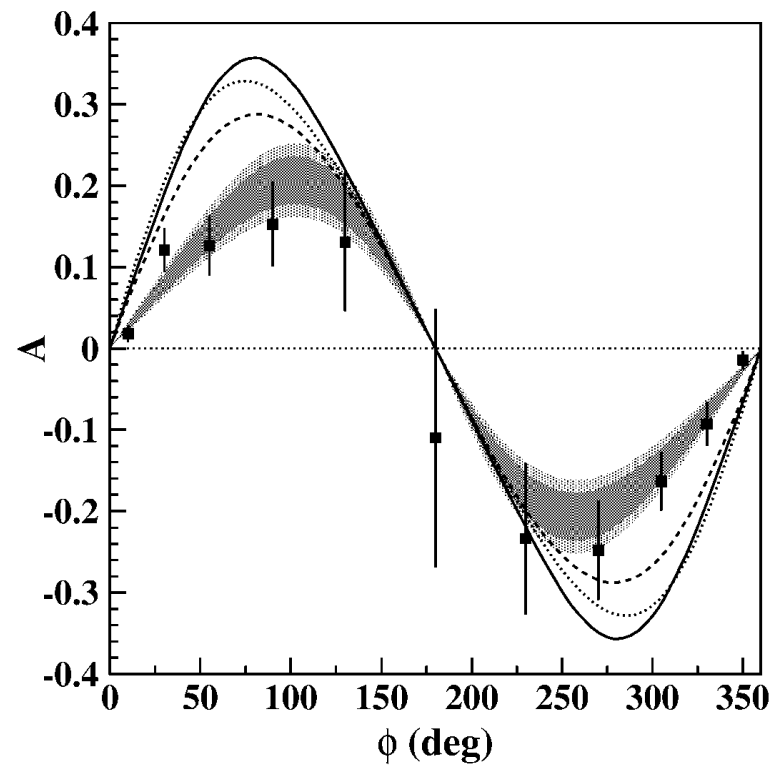

FIG. 4. $\phi$ dependence of the beam-spin asymmetry $A$. The dark shaded region is the range of the fitted function $A(\phi)$ defined by the statistical errors of parameters $\alpha$ and $\beta$, the light shaded region includes systematic uncertainties added linearly to the statistical uncertainties. The curves are model calculations according to Refs. $[6,11]$ and are discussed in the text.

photons (see e.g., Ref. [10]). In Fig. 4 the dark shaded region corresponds to the range of the fitted function within the statistical uncertainties of $\alpha$ and $\beta$. The light shaded region includes systematic uncertainties on these parameters, estimated using the method described above.

The resulting asymmetry is in a good agreement with a $\sin \phi$ modulation. The curves in Fig. 4 show the results of theoretical calculations from Refs. $[5,11,13]$ at fixed values of $Q^{2}=1.25(\mathrm{GeV} / c)^{2}, x_{B}=0.19$, and $-t=0.19(\mathrm{GeV} / c)^{2}$. The limited experimental information does not allow us to unambiguously extract GPDs from the measurement. Description of GPDs that model the $\xi$ and $t$ dependencies are therefore used to predict observables accessible in experiments. The dashed curve is a calculation at leading-twist [5] and no $\xi$ dependence in the evaluation of GPDs, the dotted curve is leading-twist with $\xi$ dependence [5], and the solid curve includes twist-3 $[14,15]$ effects. All three calculations include the $D$ term in the parametrization of the GPDs [16], which is related to double pion contributions. For a more detailed description of the model assumptions we refer to a recent review [11]. We have estimated that the model asymmetries would be reduced by about $7 \%$ if they were averaged over the experimental acceptances, bringing them somewhat closer to the measured data points.

Although the experimental results are close to the lower range of theoretical predictions, none of the calculations is in agreement with our data. This could be due to several factors which need to be studied in future research. First, parametrizations of the $\xi$ dependence of the GPDs 
were modeled in only a few different ways. Second, higher order perturbative QCD corrections have not been included. They tend to reduce the measured asymmetry [17]. Thirdly, it is possible that the average $Q^{2}$ and $W$ of our measurements are not sufficiently high to fully reach the Bjorken regime, so that some nonperturbative corrections may be needed. This can be tested in a future experiment by measuring DVCS at the same $x_{B}$ and $t$ but at higher $Q^{2}$ and $W$. Such experiments have been approved and are currently in preparation $[18,19]$.

In conclusion, we have presented the first measurement of the beam-spin asymmetry in exclusive electroproduction of real photons in the deeply inelastic regime. We see a clear asymmetry, as expected from the interference of the DVCS and BH processes [Eq. (1)]. Our results agree in sign, and are not far in magnitude to predictions based on available models of GPD parametrizations. This strongly supports expectations that DVCS will allow access to GPDs at relatively low energies and momentum transfers. This opens up a new avenue for the study of nucleon structure, which is inaccessible in inclusive scattering experiments. Further measurements at higher beam energy are planned, which will allow significant expansion of the $Q^{2}$ and $x_{B}$ range covered in these studies. The high luminosity available for these measurements will make it possible to map out details of the $Q^{2}, x_{B}$, and $t$ dependences of GPDs.

We acknowledge the outstanding efforts of the staff of the Accelerator Division and the Physics Divisions, and the Hall B technical staff that made this experiment possible. We also acknowledge useful discussions with A. Radyushkin. Also many thanks to M. Vanderhaeghen and L. Mossé for help in the calculations. This work was supported in part by the Istituto Nazionale di Fisica Nucleare, the French Centre National de la Recherche Scientifique, the French Commissariat à l'Energie Atomique, the U.S. Department of Energy, the National Science Foundation, and the Korean Science and Engineering Foundation. The Southeastern Universities Research Association (SURA) operates the Thomas Jefferson National Accelerator Facility for the United States Department of Energy under Contract No. DE-AC05-84ER40150.
*Present address: Systems Planning and Analysis, 2000 North Beauregard Street, Suite 400, Alexandria, Virginia 22311.

†Present address: Department of Physics, University of New Hampshire, Durham, New Hampshire 03824.

*Present address: Thomas Jefferson National Accelerator Facility, Newport News, Virginia 23606.

${ }^{\S}$ Present address: Department of Physics, Catholic University of America, Washington, D.C. 20064.

"Present address: The Motley Fool, Alexandria, Virginia 22314.

[1] X. Ji, Phys. Rev. Lett. 78, 610 (1997); Phys. Rev. D 55, 7114 (1997).

[2] A. V. Radyushkin, Phys. Lett. B 380, 417 (1996); Phys. Rev. D 56, 5524 (1997).

[3] J. C. Collins, L. Frankfurt, and M. Strikman, Phys. Rev. D 56, 2982 (1997).

[4] D. Müller et al., Fortschr. Phys. 42, 101 (1994).

[5] M. Vanderhaeghen, P. A. M. Guichon, and M. Guidal, Phys. Rev. Lett. 80, 5064 (1998).

[6] M. Vanderhaeghen, P. A. M. Guichon, and M. Guidal, Phys. Rev. D 60, 094017 (1999).

[7] B. Mecking et al. (to be published).

[8] The HERMES Collaboration, A. Airapetian et al., hep-ex/ 0106068 .

[9] The H1 Collaboration, C. Adloff et al., hep-ex/0107005.

[10] M. Diehl et al., Phys. Lett. B 411, 193 (1997).

[11] K. Goeke, M. V. Polyakov, and M. Vanderhaeghen, hep-ph/0106012.

[12] V. Burkert, L. Elouadrhiri, and S. Stepanyan, CLAS Analysis Note 2001-006; www.jlab.org/Hall-B/pubs

[13] M. Vanderhaeghen, P. A. M. Guichon, and M. Guidal (private communication).

[14] N. Kivel, M. V. Polyakov, and M. Vanderhaeghen, Phys. Rev. D 63, 114014 (2001).

[15] A. V. Belitsky, A. Kirchner, D. Müller, and A. Schäfer, Phys. Lett. B 510, 117 (2001).

[16] M. V. Polyakov and C. Weiss, Phys. Rev. D 60, 114017 (1999).

[17] A. Freund and M. McDermott, hep-ph/0106124.

[18] V. Burkert, L. Elouadrhiri, M. Garçon, and S. Stepanyan, JLAB Proposal PR-01-113, PAC-20; www.jlab.org/ exp_prog/generated/hallb.html

[19] Y. Roblin and F. Sabatié, JLAB Proposal E-00-110, PAC-19; www.jlab.org/exp_prog/generated/halla.html 